\title{
Depot injectable biodegradable nanoparticles loaded with recombinant human bone morphogenetic protein-2: preparation, characterization, and in vivo evaluation
}

\author{
This article was published in the following Dove Press journal: \\ Drug Design, Development and Therapy \\ 13 July 2015 \\ Number of times this article has been viewed
}

\author{
Ali Habiballah Hassan' \\ Khaled Mohamed Hosny ${ }^{2,3}$ \\ Zuahir A Murshid' \\ Adel Alhadlaq ${ }^{4}$ \\ Ahmed Alyamani ${ }^{5}$ \\ Ghada Naguib ${ }^{6}$ \\ 'Department of Orthodontics, \\ Faculty of Dentistry, ${ }^{2}$ Department \\ of Pharmaceutics and Industrial \\ Pharmacy, Faculty of Pharmacy, King \\ Abdulaziz University, Jeddah, Saudi \\ Arabia; ${ }^{3}$ Department of Pharmaceutics \\ and Industrial Pharmacy, Faculty of \\ Pharmacy, Beni Suef University, Beni \\ Suef, Egypt; ${ }^{4}$ Department of Pediatric \\ Dentistry and Orthodontics, College \\ of Dentistry, King Saud University, \\ Riyadh, ${ }^{5}$ Department of Oral Surgery, \\ ${ }^{6}$ Department of Restorative Dentistry, \\ Faculty of Dentistry, King Abdulaziz \\ University, Jeddah, Saudi Arabia
}

Objective: The aim of this study is to utilize the biocompatibility characteristics of biodegradable polymers, viz, poly lactide-co-glycolide (PLGA) and polycaprolactone (PCL), to prepare sustained-release injectable nanoparticles (NPs) of bone morphogenetic protein-2 (BMP-2) for the repair of alveolar bone defects in rabbits. The influence of formulation parameters on the functional characteristics of the prepared NPs was studied to develop a new noninvasive injectable recombinant human BMP-2 (rhBMP-2) containing grafting material for the repair of alveolar bone clefts.

Materials and methods: BMP-2 NPs were prepared using a water-in-oil-in-water doubleemulsion solvent evaporation/extraction method. The influence of molar ratio of PLGA to PCL on a suitable particle size, encapsulation efficiency, and sustained drug release was studied. Critical size alveolar defects were created in the maxilla of $24 \mathrm{New}$ Zealand rabbits divided into three groups, one of them treated with $5 \mu \mathrm{g} / \mathrm{kg}$ of rhBMP-2 NP formulations.

Results: The results found that NPs formula prepared using blend of PLGA and PCL in 4:2 $(\mathrm{w} / \mathrm{w})$ ratio showed the best sustained-release pattern with lower initial burst, and showed up to $62.7 \%$ yield, $64.5 \%$ encapsulation efficiency, $127 \mathrm{~nm}$ size, and more than $90 \%$ in vitro release. So, this formula was selected for scanning electron microscope examination and in vivo evaluation. Histomorphometric analysis showed $78 \%$ trabecular bone fill, mostly mature bone in the defects treated with rhBMP-2 in NPs within 6 weeks.

Conclusion: The prepared NPs prolonged the release and the residence time of rhBMP-2 in rabbits, which led to the formation of adequate bone in critical size alveolar bone defects in 6 weeks. This noninvasive method has application for the primary restoration of alveolar bone defects.

Keywords: BMP-2, biodegradable polymers, nanoparticle, PLGA, PCL, alveolar defects

\section{Introduction}

Bone morphogenetic proteins (BMPs) are members of the transforming growth factor- $\beta$ superfamily that act as osteoinductive factors by inducing the differentiation of osteoblasts from mesenchymal cells. ${ }^{1}$ Previous studies have shown the ability of BMPs to induce bone formation in a variety of models having many clinical applications in orthopedics and in oral and maxillofacial/dentistry, ${ }^{2-5}$ Delivery systems for growth factors with, for example, biodegradable carriers, are used to achieve slow and controlled release of the growth factor at the site of implantation. ${ }^{4}$ These include collagen, tricalcium phosphate, lactideglycolide copolymers, ehyleneglycol-lactic acid copolymer, and gelatin hydrogels., ${ }^{2,4}$
Correspondence: Ali Habiballah Hassan Department of Orthodontics, Faculty of Dentistry, King Abdulaziz University, PO Box 80209, Jeddah, 21589, Saudi Arabia Email aakbr@kau.edu.sa 
Nanotechnology deals with the utilization of materials at nanoscale level. Current research in the field of nanotechnology is attracting countries and large companies to invest in this rapidly growing area. ${ }^{9}$ Materials at nanometer scale exploit novel physical, chemical, and biological properties that can change or enhance drug properties. Because of their good biocompatibility and controlled degradation, polymeric nanoparticles (NPs) have been receiving a great deal of interest as devices for advanced drug delivery system (DDS). Polymeric nanoparticulate products provide improved delivery methods in comparison with conventional dosage forms, for several types of drugs including peptides, small molecules, nucleic acids, and proteins. ${ }^{10}$

Various factors control the drug release from NPs including the type of the polymer, physical and chemical properties, the presence of stabilizer, and type of formulation. ${ }^{11}$ The methods of preparation of the NPs largely depend on type of polymer and the solubility of the chosen drug. Variables that govern the release of the drug include chemistry of polymer, type of conjugation between polymer and drug, solubility of drug, difference in formulation parameters, and particle size and distribution in the body. ${ }^{12}$ Several techniques have been used for the production of NPs, for example, double-emulsion technique, phase separation, and spray drying. ${ }^{13}$

Biodegradable alpha-hydroxy polymer and esters polymer, particularly the copolymer of poly lactide-co-glycolide (PLGA) and polycaprolactone (PCL), are widely used in the field of controlled drug delivery. ${ }^{14}$ The use of biodegradable polymers in such systems provides an added asset, due to their enhanced tissue biocompatibility ${ }^{15}$ and the lack of necessity for surgical removal, thus improving patient compliance. ${ }^{16}$ The fabrication of such systems in the form of NPs enhances powder flowability and minimizes the pain associated with device implantation. In addition, sustainedrelease parenteral DDSs provide advantages when compared to conventional drug delivery. These advantages include (a) increased bioavailability, (b) the possibility to accurately control the resulting drug release rates over prolonged periods of time, following single administration, and (c) in addition to localized drug delivery, minimization of undesired effects of the drug. ${ }^{15}$

The objectives of this work were the following: 1) Prepare a sustained-release injectable dosage form by encapsulating the recombinant human bone morphogenetic protein-2 (rhBMP-2) into biodegradable polymers, PLGA and PCL, as NPs. 2) Study the influence of the formulation parameters on the characteristics of the prepared NPs. This is an essential step to obtain NPs formulation, with suitable particle size, high encapsulation efficiency, and sustained drug release over a long period, with minimal burst release. 3) Develop a new noninvasive procedure for the repair of surgically created alveolar bone clefts using rhBMP-2 prepared as injectable NPs.

\section{Materials and methods Materials}

rhBMP-2 was purchased from Creative Biomart (New York, NY, USA), PLGA Resomer RG $502 \mathrm{H} \mathrm{Mw} 17$ kDa was purchased from Boehringer Ingelheim (Biberach, Germany). Polycaprolactone (PCL) (molecular weight $45 \mathrm{kDa}$ ) and PLGA were purchased from Sigma/Aldrich (St. Louis, Missouri, USA). Dichloromethane was purchased from Fisher Scientific (Loughborough, UK). Polyvinyl alcohol (PVA) was purchased from Fluka Chemie GmbH (Seelze, Germany). All chemicals were of analytical grade.

\section{Methods}

\section{Preparation of BMP-2 NPs}

NPs were formed using a water-in-oil-in-water (w/o/w) double-emulsion method. An aqueous solution of rhBMP-2 was added to five solutions of PLGA:PCL (1:1, 2:1, 3:1, $4: 1$, and 5:1) in dichloromethane. The concentration of protein in the NP solution was $100 \mu \mathrm{g} / \mathrm{mL}$ of BMP-2. The polymer mixture was used at a mass of $1 \mathrm{~g}$ in $5 \mathrm{~mL}$ of dichloromethane. These phases were sonicated with probe sonicator for 3 minutes to form a water-in-oil emulsion. This emulsion was added to $200 \mathrm{~mL}$ of aqueous solution of $0.3 \mathrm{vol} \%$ PVA and sonicated for 2 minutes. This double emulsion was stirred magnetically for 4 hours at $300 \mathrm{rpm}$ before the NPs were recovered by centrifugation, washed, and lyophilized.

\section{Characterization of BMP-2 NPs}

Particle size and zeta potential determination

The prepared NPs were assayed for particle size and zeta potential analysis using Microtrac ${ }^{\circledR}$ particle size analyzer. To test the droplet size of the NPs, $10 \mathrm{mg}$ of each formulation was diluted with $10 \mathrm{~mL}$ of distilled water. The mean droplet size of the resulting dispersion was determined by dynamic light scattering using a Zetatrac machine from Microtrac, Inc. (Montgomeryville, PA, USA). The average of hydrodynamic particle size was expressed using three replicate samples.

\section{Determination of rhBMP-2-loading efficiency}

and encapsulation efficiency

The total rhBMP-2 in the prepared NPs was determined by dissolving $1 \mathrm{~mL}$ of the prepared NPs suspension in $0.1 \mathrm{~N}$ $\mathrm{NaOH}$ solution, and then, $0.1 \mathrm{~N} \mathrm{HCl}$ solution was added 
to aggregate the polymer. The acidified solution was then filtered through $0.22 \mu \mathrm{m}$ filters and assayed for rhBMP-2 content using enzyme-linked immunosorbent assay (ELISA). Encapsulation efficiency of rhBMP-2 was determined by the following equation:

$$
\mathrm{EE} \%=W_{\mathrm{E}} / W_{\mathrm{T}} \times 100
$$

where $W_{\mathrm{E}}$ is the weight of rhBMP-2 entrapped within the prepared NPs and $W_{\mathrm{T}}$ is the total weight of rhBMP-2 used during the preparation. Loading efficiency (LE\%) was determined by the following equation:

$$
\mathrm{LE} \%=W_{\mathrm{E}} / W_{\mathrm{p}} \times 100
$$

where $W_{\mathrm{E}}$ is the weight of rhBMP-2 entrapped within the prepared NPs and $W_{\mathrm{p}}$ is the total weight of NPs.

\section{In vitro release study}

The in vitro drug release tests were determined by the dialysis method. That is, rhBMP-2 NPs were placed into a dialysis bag (molecular weight cut-off of $45 \mathrm{kDa}$ ), and the test was carried out using the USP Dissolution Apparatus II fixed with rotating baskets immersed in phosphate buffer $\mathrm{pH}$ 6.8. Samples of $5 \mathrm{~mL}$ were withdrawn after different time intervals and replaced with an equal volume of fresh media. The samples were filtered through $0.45 \mu \mathrm{m}$ filters and assayed for protein in the rhBMP-2 by spectrophotometry at $280 \mathrm{~nm}$. Each test was carried out in triplicate. According to the results of NPs characterization and in vitro release, the selected formula was chosen for further scanning electron microscopy (SEM) examination and in vivo evaluation.

\section{Examination of NPs morphology}

According to the results of NPs characterization and in vitro release, the selected formula F3 was chosen for examination by using SEM (Jeol JSM-5400 LV, Jeol Ltd, Tokyo, Japan). F3 NPs formulation was spread on a layer of double-adhesive tape on a metal SEM stub and gold coated using a sputter coating device for examination of NP morphology.

\section{The in vivo study}

A total of 24, 7-week-old, male, New Zealand White rabbits ranging from $2.5 \mathrm{~kg}$ to $3.0 \mathrm{~kg}$ in weight were used. The surgical procedure to create experimental alveolar clefts was performed according to the method described in a previous study in which BMP-2 was prepared as in situ liposomal hydrogel. ${ }^{8}$ A $10 \mathrm{~mm} \times 5 \mathrm{~mm} \times 5 \mathrm{~mm}$ osseous defect was created on both sides of the maxillary bone, approximately $5 \mathrm{~mm}$ distal to the incisor, and left to heal. The rabbits then received the assigned solution by subcutaneous injection. The animals were divided into three groups according to the treatment they received: group A injected with $5 \mu \mathrm{g} / \mathrm{kg}$ of rhBMP-2 in isotonic saline (positive control), group B injected with isotonic saline without rhBMP-2 (negative control), and group C injected with $5 \mu \mathrm{g} / \mathrm{kg}$ of rhBMP-2 in the assigned NPs suspension formulation F3 prepared using blend of PLGA and PCL in 4:2 (w/w) ratio. For pharmacokinetic evaluation, plasma samples were taken from rabbits in the three groups, before the injection and after 1 hour, 2 hours, 3 hours, 6 hours, 12 hours, 24 hours, 2 days, and 4 days. All samples were analyzed for rhBMP-2 content using an ELISA.

All animals were kept in an ideal controlled environment and were fed a standard diet, and their general health was monitored daily. ${ }^{8}$ After 6 weeks, the rabbits were euthanized using $\mathrm{CO}_{2}$ according to the regulations of King Abdulaziz University Animal Care Committee. Maxillae were dissected, processed for light microscopy, and stained for histological assessment. Histologic sections were assessed under light microscopy. Histomorphometric analysis was also performed to quantitate the total bone volume produced in each of the created defects. ${ }^{8}$ Multiple comparisons among the groups were performed by one-way analysis of variance followed by Tukey's multiple comparison test using SPSS statistical package version 16 (SPSS, Chicago, IL, USA). The level of significance was set in advance at $5 \%$.

\section{Results}

The prepared NPs were characterized in terms of particle size, zeta potential, loading and encapsulation efficiency, and morphology. The effect of PLGA-to-PCL ratio on the characteristics of NPs is shown in Table 1. All NPs showed a particle size ranging from $83 \mathrm{~nm}$ to $190 \mathrm{~nm}$. The particle size is an important parameter, as it can influence the biopharmaceutical properties and the fate of NPs. ${ }^{11}$ The particle size was $83 \mathrm{~nm}$ in case of NPs prepared in PLGA alone. By increasing the ratio of PCL relative to PLGA, the formed NPs showed a significantly larger $(P<0.05)$ mean diameter (for PLGA:PCL at ratio 4:4, the size was $190 \mathrm{~nm}$ ). The results of zeta potential measurements indicated that zeta potentials became more negative as more PCL was added. All prepared NP formulations had zeta potentials between $-18 \mathrm{mV}$ and $-24.5 \mathrm{mV}$ (Table 1).

SEM photos of the prepared NPs for the different formulae showed spheres with smooth surfaces (Figure 1) with 
Table I Particle size, zeta potential, LE\%, and EE\% of different rhBMP-2-prepared nanoparticles

\begin{tabular}{llllll}
\hline Formula & PLGA:PCL ratio & Particle size $(\mathbf{n m})$ & Zeta potential $(\mathbf{m V})$ & LE\% & EE\% \\
\hline FI & $4: 0$ & $83 \pm 4$ & -18.9 & $57.3 \pm 4.32$ & $41.4 \pm 3.11$ \\
F2 & $4: 1$ & $110 \pm 8$ & -20.2 & $59.6 \pm 6.11$ & $52.6 \pm 4.93$ \\
F3 & $1: 2$ & $127 \pm 7$ & -22.3 & $62.7 \pm 5.94$ & $64.5 \pm 5.33$ \\
F4 & $151 \pm 9$ & -23.9 & $63.1 \pm 5.22$ & $67.2 \pm 4.27$ \\
F5 & $4: 4$ & $190 \pm I I$ & -24.5 & $66.4 \pm 6.21$ & $65.5 \pm 5.09$ \\
\hline
\end{tabular}

Abbreviations: LE, loading efficiency; EE, encapsulation efficiency; rhBMP-2, recombinant human bone morphogenetic protein-2; PLGA, poly lactide-co-glycolide; PCL, polycaprolactone.

average diameter close to the measured diameter obtained using light scattering technique (zetasizer).

The cumulative percentages of rhBMP-2 released from NPs based on different PLGA:PCL ratios as a function of time are shown in Figure 2. The slowest release was obtained at a PLGA:PCL ratio of $4: 4$, but the cumulative percentage of BMP-2 released at the end of the test was $60 \%$ only. Formula F3 prepared by PLGA:PCL at ratio 4:2 released BMP-2 in controlled manner, and the release pattern kinetically followed Higuchi diffusion model and at same time showed high percentage of BMP-2 release, and hence, this formula was selected for in vivo study.

Histological and histomorphometrical analysis showed significantly more bone formation in group $\mathrm{C}$ when compared to groups A and B (Table 2). In group A, rhBMP-2 injected with isotonic saline, plasma levels of BMP-2 measured by ELISAs rapidly increased during the first 6 hours to more than $20 \mu \mathrm{g} / \mathrm{L}$ and then decreased to less than $5 \mu \mathrm{g} / \mathrm{L}$ within 48 hours (Figure 3). This result is in accordance with the histological examination, which revealed that there were few

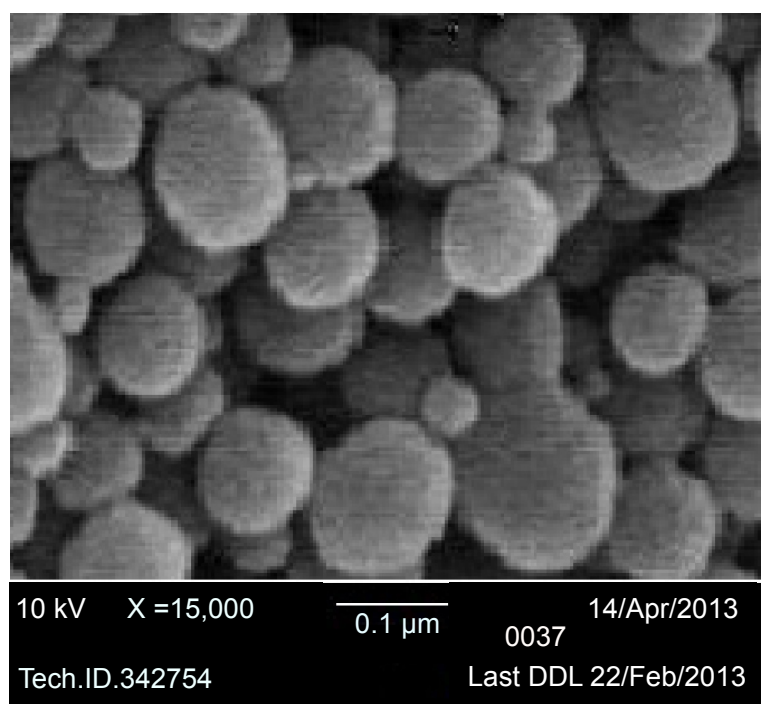

Figure I SEM images of rhBMP-2 nanoparticles.

Abbreviations: SEM, scanning electron microscopy; rhBMP-2, recombinant human bone morphogenetic protein-2; DDL, Dynamic data links. bony spicules embedded in a fibrous tissue filling the defect (Figure 4). Histomorphometrically, the repaired defects in group A were on average 19\% ossified (Table 2). In group B (negative control), plasma levels of rhBMP-2 were untraceable during the entire 96-hour period of release (Figure 5). This is in accordance with the histological examination (Figure 6A and B), which revealed very few bone spicules in some slides and complete absence of any newly formed bone in others (Table 2), with mostly fibrous tissue filling the whole created defects. In group C (rhBMP-2 in NPs), the plasma level of rhBMP-2 slowly increased to $16 \mu \mathrm{g} / \mathrm{L}$ within 48 hours. After 48 hours, the plasma level did not decrease significantly but remained at a relatively steady plateau during the 4-day test period (Figure 7). This is in accordance with the histological examination, which showed extensive active bone formation surrounding the NPs, which were not yet completely resorbed. In general, the newly formed bone was mature with many distinct osteocytes. Multiple osteoblasts and blood vessels were seen all over the defects (Figure $8 \mathrm{~A}-\mathrm{C}$ ). The newly formed bone volume was $78 \%$.

\section{Discussion}

The w/o/w double-emulsion solvent evaporation/extraction method that was used in the present study appeared to be a

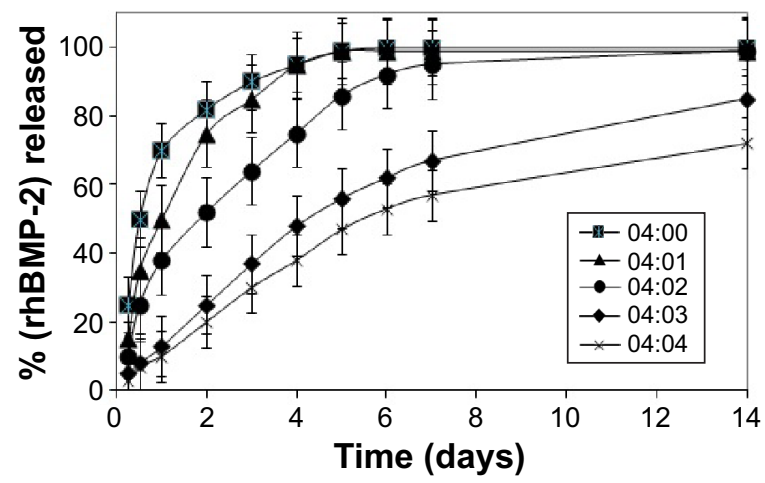

Figure 2 Release of rhBMP-2 from nanoparticles prepared with various PLGA:PCL ratios.

Abbreviations: rhBMP-2, recombinant human bone morphogenetic protein-2; PLGA, poly lactide-co-glycolide; PCL, polycaprolactone. 
Table 2 Comparison of total bone volume measured in the created bone defects using one-way ANOVA and Tukey's multiple comparisons

\begin{tabular}{llllll}
\hline Group & $\begin{array}{l}\text { Number of } \\
\text { animals/slides }\end{array}$ & Nanoparticles & $\begin{array}{l}\text { rhBMP-2 } \\
(\mu \mathrm{g} / \mathbf{k g})\end{array}$ & $\begin{array}{l}\text { Concentration of BMP } \\
\text { at 96 hours }(\mu \mathrm{g})\end{array}$ & $\begin{array}{l}\% \text { of the new } \\
\text { bone volume } \pm \text { SD }\end{array}$ \\
\hline A & $8 / 28$ & - & 5 & $<2$ & $19 \pm 3^{\mathrm{a}}$ \\
B & $8 / 28$ & - & - & 0 & $12 \pm 4^{\mathrm{a}}$ \\
$\mathrm{C}$ & $7 / 28$ & + & 5 & 11 & $78 \pm 4^{\mathrm{b}}$ \\
\hline
\end{tabular}

Notes: Groups identified by different superscript letters are significantly different $(P<0.05)$ by one-way ANOVA and Tukey's multiple comparisons. $-=$ no, $+=$ yes. Abbreviations: ANOVA, analysis of variance; rhBMP-2, recombinant human BMP-2; BMP, bone morphogenetic protein; SD, standard deviation.

suitable technique to formulate rhBMP-2-loaded NPs based on PLGA and its PCL copolymers. Moreover, although the use of PVA as emulsifier can make uniform particles with small size, it has been recently demonstrated that it can form an interconnected network with the PLGA polymer at the interface that would compromise the dispersibility of the NPs. ${ }^{17}$

Regarding the encapsulation and loading efficiencies, the results indicated that incorporation of PCL as a copolymer with PLGA increased the entrapment of the drug due to the polymer phase becoming more viscous and forming more rigid NPs that entrap more rhBMP-2 within the particles. Furthermore, PCL copolymers generate a polymer network characterized by a more irregular and disordered crystalline structure that seems to promote the accommodation of more rhBMP-2 molecules. These results were in agreement with previous studies, which included encapsulation of docetaxel in PLGA NPs. ${ }^{18}$ The increase in entrapment and loading efficiency by increasing the PCL ratio occurred up to 4:3, but with further increase in PCL (PLGA:PCL at 4:4), the EE\% decreased. This may be due to the fact that at higher PCL ratios, a fast precipitation of polymer occurs after addition of the organic phase to the surfactant solution.

The zeta potential results indicated that there was a slight effect of increase in the PLGA:PCL ratio on the surface

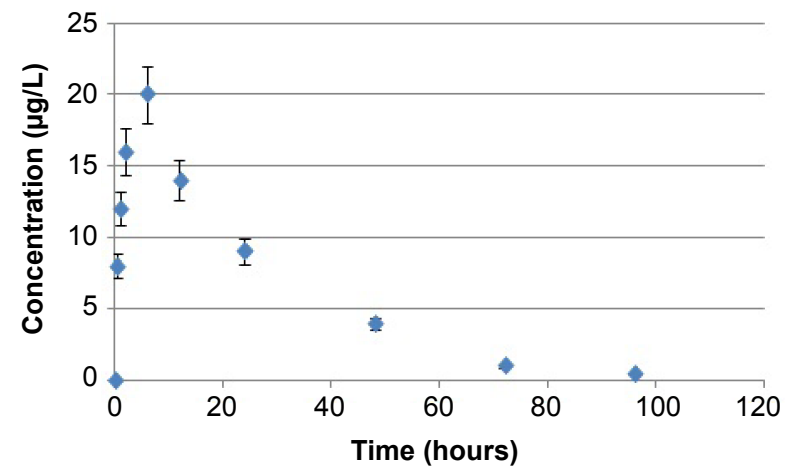

Figure 3 Plasma levels of BMP-2 after local injection of $5 \mu \mathrm{g} / \mathrm{kg}$ of BMP-2 isotonic saline into the created alveolar bone defects in rabbits (group A).

Abbreviation: BMP-2, bone morphogenetic protein- 2 . charge of NPs. All prepared NP formulations had zeta potential between $18.9 \mathrm{mV}$ and $24.5 \mathrm{mV}$. When all NPs have the same negative charge, they tend to remain stable and resist aggregation by electrostatic repulsion.

The release rate of rhBMP-2 from the NPs was strongly affected by the PLGA:PCL ratio of the polymer matrix. In particular, PLGA-based NPs are characterized by the higher initial burst percentage during the first day of the release (approximately $70 \%$ of rhBMP- 2 is released within 24 hours) that is attributed to the presence of the hydrophilic glycolide units in the PLGA polymer causing an initial release. The higher hydrophilicity of the polymer improved water permeability into NPs causing a more rapid drug release as well as a faster degradation of the polymer. ${ }^{2}$ This was probably responsible for the high initial rhBMP-2 release. By increasing the ratio of PCL in the polymer matrix, the burst percentage decreased to $10 \%$, and $13 \%$ released only during 24 hours in case of NPs prepared with polymer matrix formed from PLGA:PCL in ratios 4:4 and 4:3, respectively. This could be attributed to the increase in stiffness of the NPs formed with PLGA-PCL mixture and to the higher viscosity of the polymeric solution, which leads to formation of NPs with longer diffusion pathways that retard the release of rhBMP-2 from the NPs. ${ }^{19}$

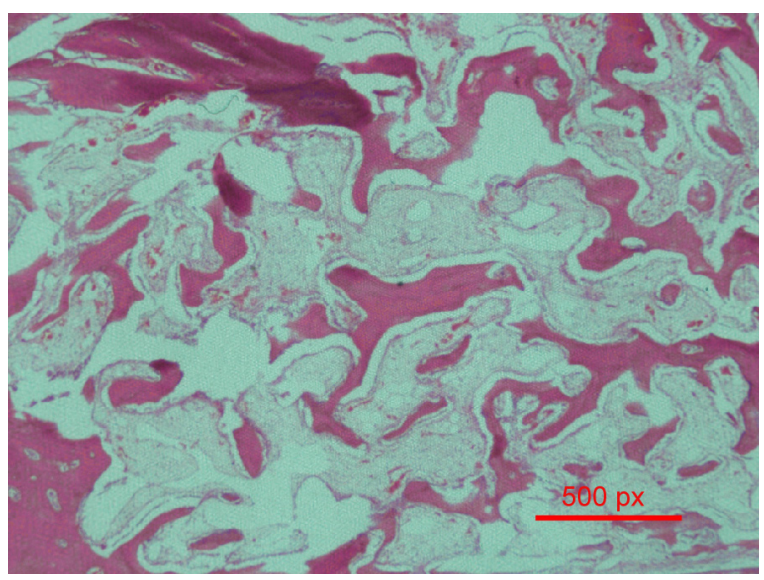

Figure 4 Photomicrograph of a bone defect in group A (H\&E stain, at $4 \times$ magnification).

Abbreviations: H\&E, hematoxylin and eosin; px, pixels. 


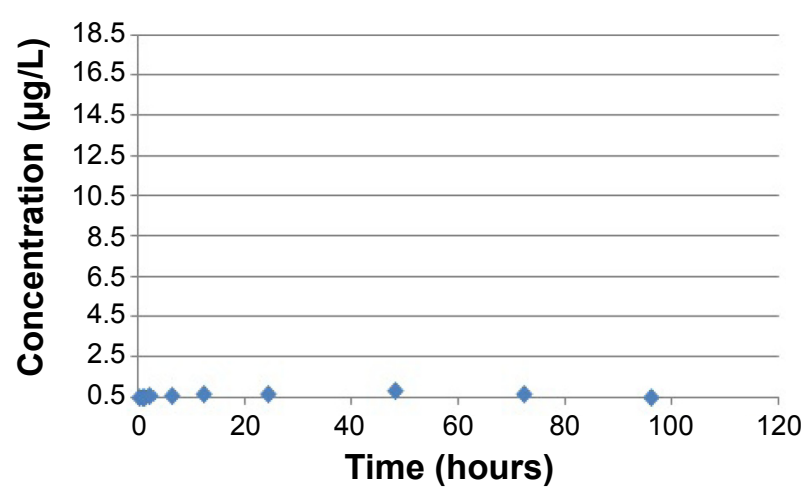

Figure 5 Plasma level of BMP-2 after injection of isotonic saline into the created defects in the rabbits (group B).

Abbreviation: BMP-2, bone morphogenetic protein-2.

Histologically, in groups A and B, few bony spicules were formed. This could be explained by the fact that in group A, the rhBMP-2 was dissolved in isotonic saline only, while in group B, no rhBMP-2 was used at all. This is in agreement with many previous studies, which found that the rhBMP-2 is inefficient in the absence of a controlled-release delivery system. ${ }^{20}$ In group C, NP use to deliver rhBMP-2 was optimized for the slow release of rhBMP-2, due to its availability at the defect, where the polymer was assumed to bind with soft tissue, and then release the protein in an optimum manner. This is because in this formula, the rhBMP-2 is
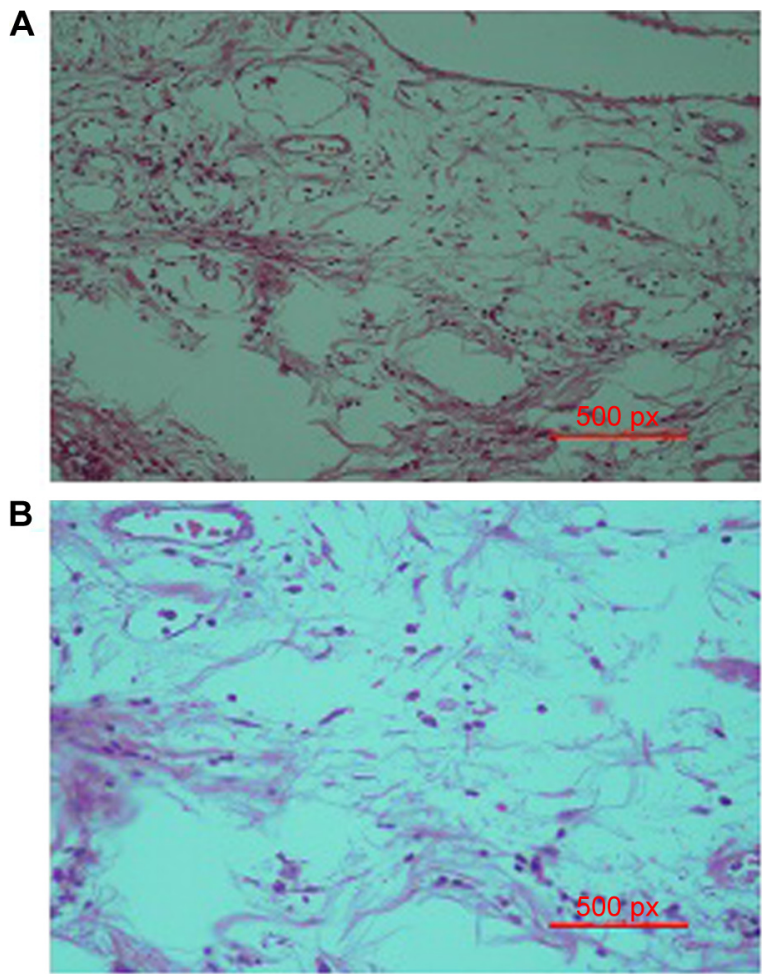

Figure 6 Photomicrographs of a bone defect in group B (slides stained with H\&E stain, at $4 \times$ magnification (A) and $10 \times$ magnification (B)).

Abbreviations: H\&E, hematoxylin and eosin; px, pixels.

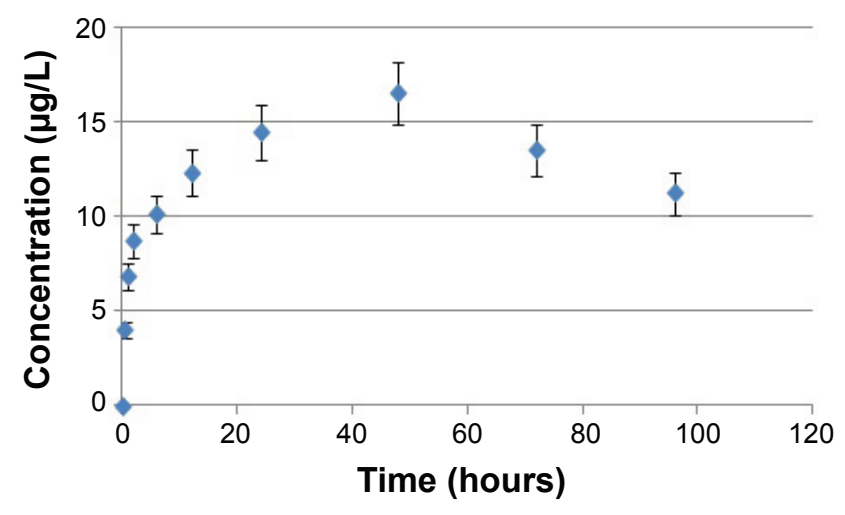

Figure 7 Plasma level of BMP-2 after injection of $5 \mu \mathrm{g} / \mathrm{kg}$ of rhBMP-2 nanoparticles into the surgically created alveolar bone defects in the rabbits (group $\mathrm{C}$ ).

Abbreviations: BMP-2, bone morphogenetic protein-2; rhBMP-2, recombinant human BMP-2.

entrapped within NPs, which retarded the release of rhBMP-2 and maintained its plasma concentration at steady state for a longer period of time.

The present study is a continuation of a previous work, ${ }^{8}$ in which a new noninvasive method of treatment was investigated, testing rhBMP-2 encapsulated within reverse evaporation phase vesicles (REVs) liposome and loaded within an in situ gel base. The maximum plasma concentration of rhBMP-2 in the current study $16.5 \mu \mathrm{g} / \mathrm{L}$ was more than twice that seen in the liposomal study $(6.5 \mu \mathrm{g} / \mathrm{L}){ }^{8}$ This suggests that the pharmacokinetics of drugs upon delivery in nano-formulations is much higher using NPs than using liposomes. ${ }^{21}$ Also, the time to reach maximum plasma level in case of liposomal in situ gel was 18 hours only, and the concentration started to decline after 18 hours. However, the NPs used in the present study successfully maintained the increased plasma rhBMP- 2 concentration for 48 hours, which indicated that the NPs prolonged and controlled the release of rhBMP-2 more than did the liposomal in situ gels. This could be due to the fact that NP formulations are generally more stable than liposomal formulations due to the solid nature of NPs. ${ }^{22}$ Finally, the newly formed bone was more mature and had a greater volume when NPs were used than when liposomal in situ gels were used. ${ }^{8}$ However, unlike the liposomal in situ gel group in our previous study, remnants of the injected NPs were observed in group $\mathrm{C}$ in the present study, which indicates that the rate of biodegradation of the NPs was lesser than that of liposomal in situ gels.

The combined results of this research suggested many future applications for this promising idea of using noninvasive, injectable rhBMP-2. One of these indications is the primary grafting of alveolar bone defects in cleft lip and palate patients, which is increasing in prevalence in many developing 

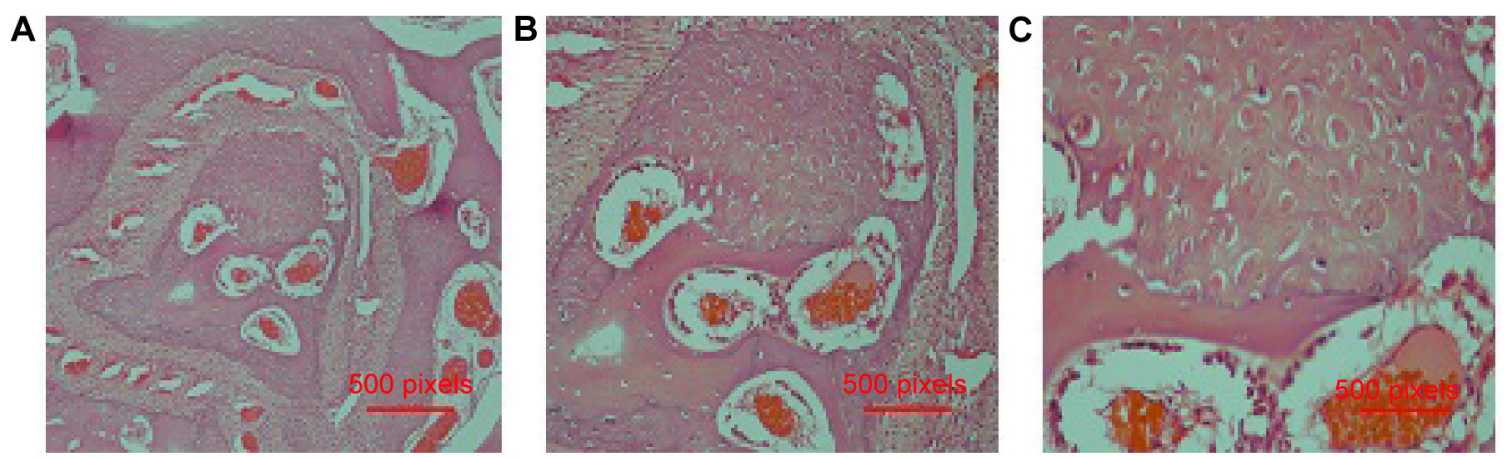

Figure 8 Photomicrographs of a bone defect in group C (slides stained with H\&E stain, at $10 \times$ magnification $(\mathbf{A}), 20 \times$ magnification $(\mathbf{B})$, and $40 \times$ magnification $(\mathbf{C})$ ). Abbreviation: H\&E, hematoxylin and eosin.

countries. ${ }^{23}$ This method should reduce the postsurgical retardation of maxillary growth that is expected with the standard invasive secondary grafting techniques in the current use, due to the lack of scarring when grafted material is introduced by injection. However, further standardization and trials of such methods are required before human use can proceed.

\section{Conclusion}

Encapsulation of rhBMP-2 within PLGA/PCL NPs is a DDS that seems to provide superior new noninvasive procedure to repair alveolar cleft defects. The defects treated with rhBMP-2 within PLGA/PCL NPs were found to be $78 \%$ ossified with surrounding fibro-vascular tissues containing many osteoblasts within 6 weeks. This novel method has many potential future applications, especially as an early grafting method for alveolar bone defects in cleft lip and palate patients.

\section{Acknowledgments}

This research has been funded by the Deanship of Scientific Research at King Abdulaziz University through the grant number (MG/31/4) for the group entitled "Dentofacial Deformities and Cleft Lip and Palate". The authors also would like to thank Dr Aziza Alhubail and Dr Abdullah Abbaddi, Saudi Board of Orthodontics residents, for their valuable participation during the conduct of this research.

\section{Disclosure}

The authors report that there is no conflict of interest in this work.

\section{References}

1. Hassan AH, Evans CA, Zaki AM, George A. Use of bone morphogenetic protein-2 and dentin matrix protein-1 to enhance the osteointegration of the Onplant system. Connect Tissue Res. 2003;44:30-41.

2. Mori M, Isobe M, Yamazaki Y, Ishihara K, Nakabayashi N. Restoration of segmental bone defects in rabbit radius by biodegradable capsules containing recombinant human bone morphogenetic protein-2.J Biomed Mater Res. 2000;50:191-198.
3. Hassan AH, Al-Hubail A, Al-Fraidi AA. Bone inductive proteins to enhance postorthodontic stability. Angle Orthod. 2010;80:1051-1060.

4. Herford AS, Boyne PJ. Reconstruction of mandibular continuity defects with bone morphogenetic protein-2 (rhBMP-2). J Oral Maxillofac Surg. 2008;66:616-624.

5. Geiger M, Li RH, Friess W. Collagen sponges for bone regeneration with rhBMP-2. Adv Drug Deliv Rev. 2003;55:1613-1629.

6. Hibi H, Yamada Y, Ueda M, Endo Y. Alveolar cleft osteoplasty using tissue-engineered osteogenic material. Int J Oral Maxillofac Surg. 2006;35:551-555.

7. Sawada Y, Hokugo A, Nishiura A, et al. A trial of alveolar cleft bone regeneration by controlled release of bone morphogenetic protein: an experimental study in rabbits. Oral Surg Oral Med Oral Pathol Oral Radiol Endod. 2009;108:812-820.

8. Almaqboul RA, Hassan AH, Hosny KM, et al. Non-invasive method to repair alveolar bone defect. J Dent Res. 2014;93(B):711.

9. Arias LJ, Lopez-Viota M, Gallardo V, Ruiz MA. Chitosan nanoparticles as a new delivery system for the chemotherapy agent tegafur. Drug Dev Ind Pharm. 2010;36:744-750.

10. Sonaje K, Italia JL, Sharma G, Bhardwaj V, Tikoo K, Kumar MN. Development of biodegradable nanoparticles for oral delivery of ellagic acid and evaluation of their antioxidant efficacy against cyclosporine A-induced nephrotoxicity in rats. Pharm Res. 2007;24:899-908.

11. Ahlin P, Kristl J, Kristl A, Vrecer F. Investigation of polymeric nanoparticles as carriers of enalaprilat for oral administration. Int J Pharm 2002;239:113-120.

12. Dillen K, Vandervoort J, Ludwig A. Factorial design, physicochemical characterisation and activity of ciprofloxacin-loaded PLGA nanoparticles for ocular use. J Control Release. 2005;101:369-371.

13. Dong YC, Feng SS. Poly(D,L-lactide-co-glycolide)/montmorillonite nanoparticles for oral delivery of anticancer drugs. Biomaterials. 2005;26:6068-6076.

14. Hadinoto K, Cheow WS. Hollow spherical nanoparticulate aggregates as potential ultrasound contrast agent: shell thickness characterization. Drug Dev Ind Pharm. 2009;35:1167-1179.

15. Lin A, Chen J, Liu Y, et al. Preparation and evaluation of N-caproyl chitosan nanoparticles surface modified with glycyrrhizin for hepatocyte targeting. Drug Dev Ind Pharm. 2009;35:1348-1355.

16. Avgoustakis K, Beletsi A, Panagi Z, Klepetsanis P, Karydas AG, Ithakissios DS. PLGA-mPEG nanoparticles of cisplatin: in vitro nanoparticle degradation, in vitro drug release and in vivo drug residence in blood properties. J Control Release. 2002;79:123-135.

17. des Rieux A, Fievez V, Garinot M, Schneider YJ, Preat V. Nanoparticles as potential oral delivery systems of proteins and vaccines: a mechanistic approach. J Control Release. 2006;116:11-27.

18. Misara H, Salim H, Barbos D, Paunoiu C, Fessi H. Preparation and characterization of radioactive dirhenium decacarbonyl-loaded PLGA nanoparticles for radionuclide intra-tumoral therapy. Eur J Pharm Biopharm. 2007;67:597-611. 
19. Yu Y, Tan S, Zhao S, et al. Antitumor activity of docetaxel-loaded polymeric nanoparticles fabricated by shirasu porous glass membraneemulsification technique. Int J Nanomedicine. 2013;8:2641-2652.

20. Wang G, Siggers K, Zhang S, et al. Preparation of BMP-2 containing bovine serum albumin (BSA) nanoparticles stabilized by polymer coating. Pharm Res. 2008;25:2896-2909.

21. Hosny K, Aljeaid B. Sildenafil citrate as oral solid lipid nanoparticles: a novel formula with higher bioavailability and sustained action for treatment of erectile dysfunction. Expert Opin Drug Deliv. 2014;11: 1015-1022.
22. Torchilin VP. Micellar nanocarriers: pharmaceutical perspectives. Pharm Res. 2007;24:1-16.

23. Alamoudi NM, Sabbagh HJ, Innes NP, et al. Prevalence and characteristics of non-syndromic orofacial clefts and the influence of consanguinity. J Clin Pediatr Dent. 2014;38:241-246.

\section{Publish your work in this journal}

Drug Design, Development and Therapy is an international, peerreviewed open-access journal that spans the spectrum of drug design and development through to clinical applications. Clinical outcomes, patient safety, and programs for the development and effective, safe, and sustained use of medicines are a feature of the journal, which has also been accepted for indexing on PubMed Central. The manuscript management system is completely online and includes a very quick and fair peer-review system, which is all easy to use. Visit http://www.dovepress.com/testimonials.php to read real quotes from published authors.

Submit your manuscript here: http://www.dovepress.com/drug-design-development-and-therapy-journal 